\title{
An Alternate Charging Method For Additional Smart Switch In Weak Light Environment
}

\author{
Jing Wang ${ }^{1, a}$,Lijun Wang ${ }^{2, ~ b}$,Zhicheng Wang ${ }^{3 . c}$ and Junjie Wang ${ }^{4, d}$ \\ 1,2,3,4 Zhejiang University of Science and Technology, 318 Liu He road, Xihu District, \\ Hangzhou, Zhejiang, China \\ a770398296@qq.com, bdagouwang@zju.edu.cn,c17826805345@163.com,d 916925234@ qq.com
}

Keywords: alternate charging, low-light, hysteresis comparator, supercapacitor Abstract. Additional Smart Switch (ASS) is especially suitable for lighting power saving of existing buildings because its installation requires no wall damage and no rewiring. However, as a new device working in low light environment, it is a challenge for ASS to charge lithium battery with solar panels. This paper offers an alternate charging method in weak light conditions for ASS. Following this method, the chargeable battery will be charged efficiently by the way of continuous "electricity accumulating" and alternate "battery charging". Furthermore, this paper put forward a self-adaptive judgment method for determining the opening time of "battery charging". The alternate charging method provided by this paper has been validated through a series of experiments and applied to actual ASS products.

\section{Introduction}

In China, building lighting energy consumption has accounted for $13 \%$ of the total energy consumption ${ }^{[1]}$. Recent years, smart lighting switches have received wide attention for its lighting energy saving through remote shutdown that help people overcome the bad habit of leaving out without turning off the lights ${ }^{[2]}$. However, the large-scale application of smart switches in existing structures is still severely limited because it will cause wall damage and rewiring ${ }^{[3]}$.

In contrast, Additional Smart Switch (ASS for short) will be more suitable for completed buildings because it can be fastened directly on traditional switch panels ${ }^{[4]}$. Installation effect diagram of ASS is depicted in Fig 1. However, the power supply of ASS is the major challenge: battery is inconvenient owing to the fact that it needs to be changed frequently, and solar power is inefficient in one room with low light intensity ${ }^{[5]}$.

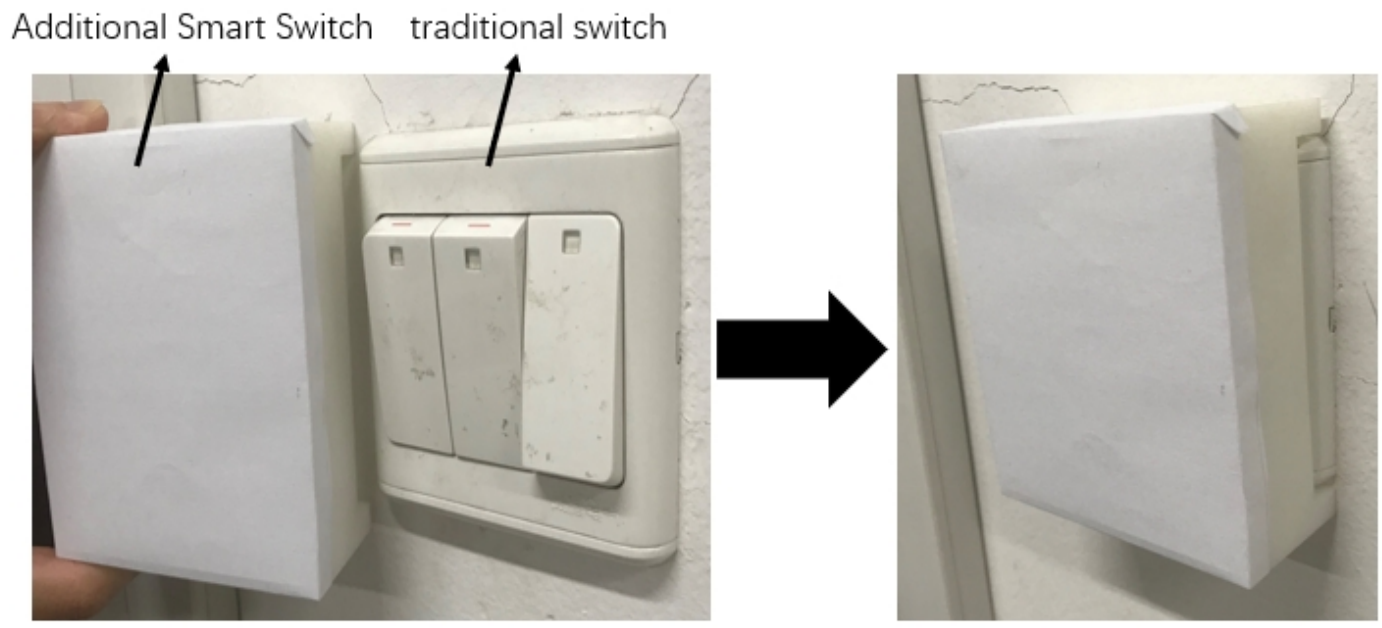

Fig 1. Installation effect diagram of ASS

This paper provides an alternate charging method for weak illumination environs based on self-adaptive judgment. Compared to traditional photovoltaic charging with low efficiency under dim light conditions, the method presented in this paper adopts a charging process of continuous "electricity accumulating" and alternate "battery charging" to achieve high charging efficiency. 
Furthermore, this paper put forward a self-adaptive judgment method for determining the opening time of "battery charging" instead of using a single chip microcomputer. The remainder of this paper offers the principle and application of alternate charging method.

\section{General Principle}

1) General principle of alternate charging method

In the condition of strong light, the alternate charging method, like traditional photovoltaic power generation, can charge electric energy generated from solar panels to a chargeable lithium battery directly. However, under the surroundings of low light, different from normal solar charging loses efficiency greatly, the alternate charging method maintains a high efficiency by dividing the charging process into two part: "electricity accumulating" and "battery charging".

Between the solar panel and the battery, the alternate method employs supercapacitor, boost module, charge management module and hysteresis comparator to form whole circuit for self-determine and switch charging process automatically. The overall configuration of the charging method is depicted in Fig 2.

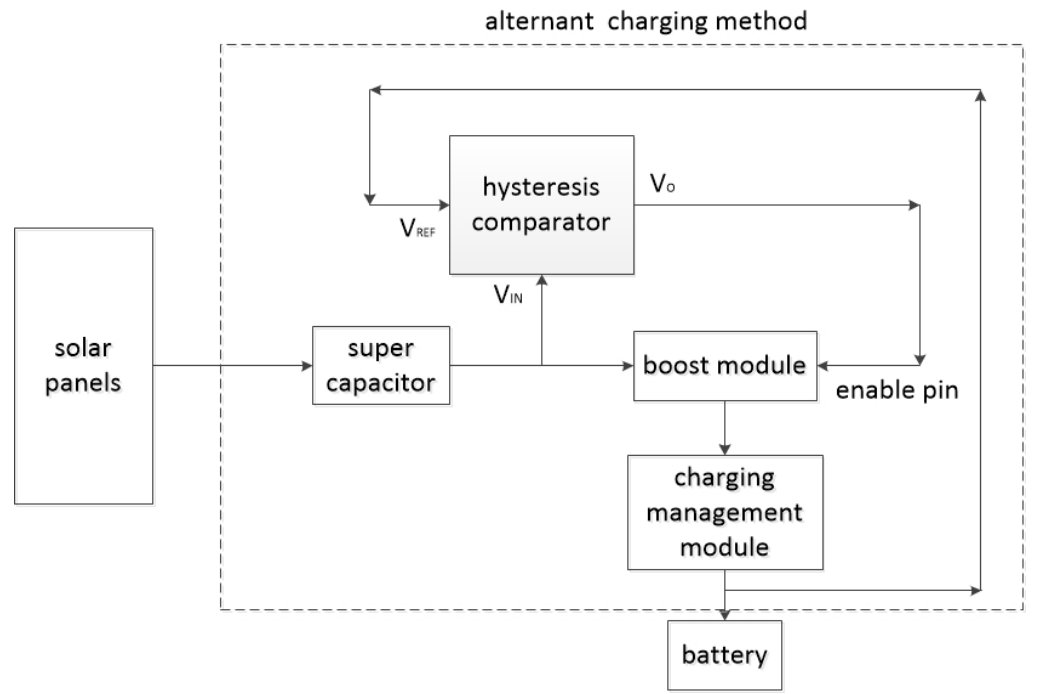

Fig 2. Charging method configuration

The hysteresis comparator attains self-adaptive judgement by dint of suppling different upper and lower threshold voltages to supercapacitor according to the voltage of the battery. The supercapacitor will enter "battery charging" only when the upper threshold voltage is reached and discharge to lower threshold voltage and "electricity accumulating" runs through the whole charging process as long as there is outside light.

Therefore, the threshold voltage corresponding to the supercapacitor is key of this method.

\section{2) Requirements for starting Alternate Charging}

The lithium battery charging is divided into three stages, the first stage is pre-charge phase, next is constant current variable voltage charging phase and last is the constant voltage charging phase ${ }^{[6]}$. In view of working conditions and characteristics of ASS, the method is mainly applied to the second stage of battery charging. Curve of charge lithium battery and curve of upper and lower threshold voltage are depicted in Fig 3. The threshold voltage is proportional to the battery voltage and it always is lower than the battery voltage because of boost module. This means that the supercapacitor has to store the corresponding electrical energy according to the different lithium battery voltage before entering "battery charging". 


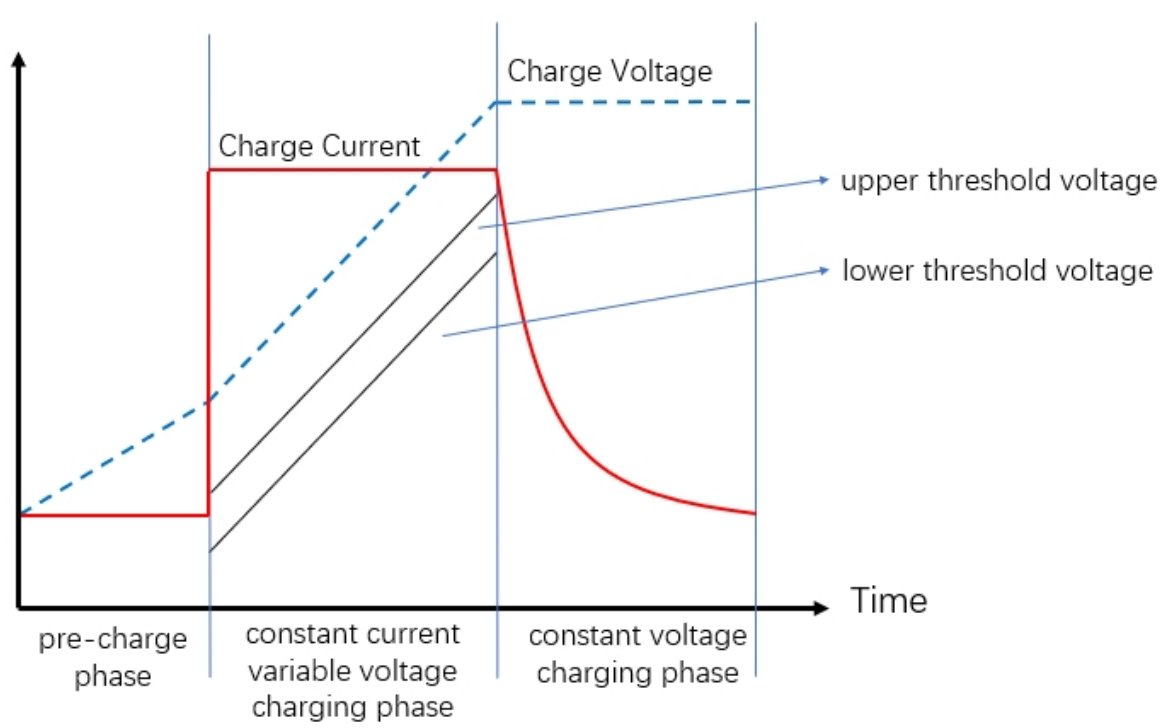

Fig 3. Curve of battery charge and threshold voltage

3) Work flow of charging method

Work flow of charging method is depicted in Fig 4. The energy generated by solar panels is stored in the supercapacitor. This is "electricity accumulating" and it is ongoing all the time. When the voltage of the supercapacitor reaches the upper threshold voltage from hysteresis comparator, "battery charging" is running and supercapacitor begins to discharge. Electric energy fills lithium battery with the help of boost module and charge management module. When the supercapacitor voltage drops to the lower threshold voltage, "battery charging" interrupts.

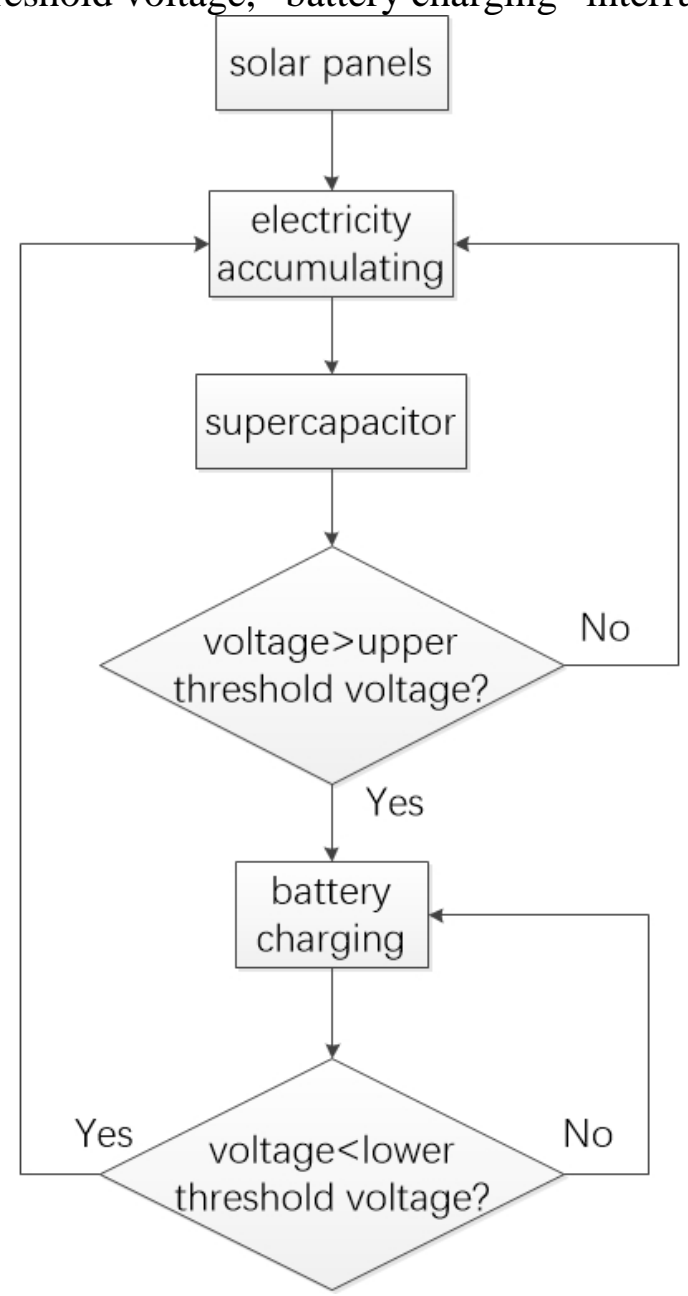

Fig 4. Work flow of charging method 


\section{Experiment And Result Discussion}

1) Design of charging circuit

a) Design of boost module

The design of the boost module for weak energy should pay attention to the energy consumption, conversion efficiency and output voltage. In this boost circuit, the energy consumption of the circuit is determined by the static current. The conversion efficiency is proportional to the reciprocal of the capacitance and it has a function relationship with the input and output voltage. Circuit of boost module and values of components are depicted in Fig 5. The output voltage is programmed by R1 and $\mathrm{R} 2^{[7]}$.

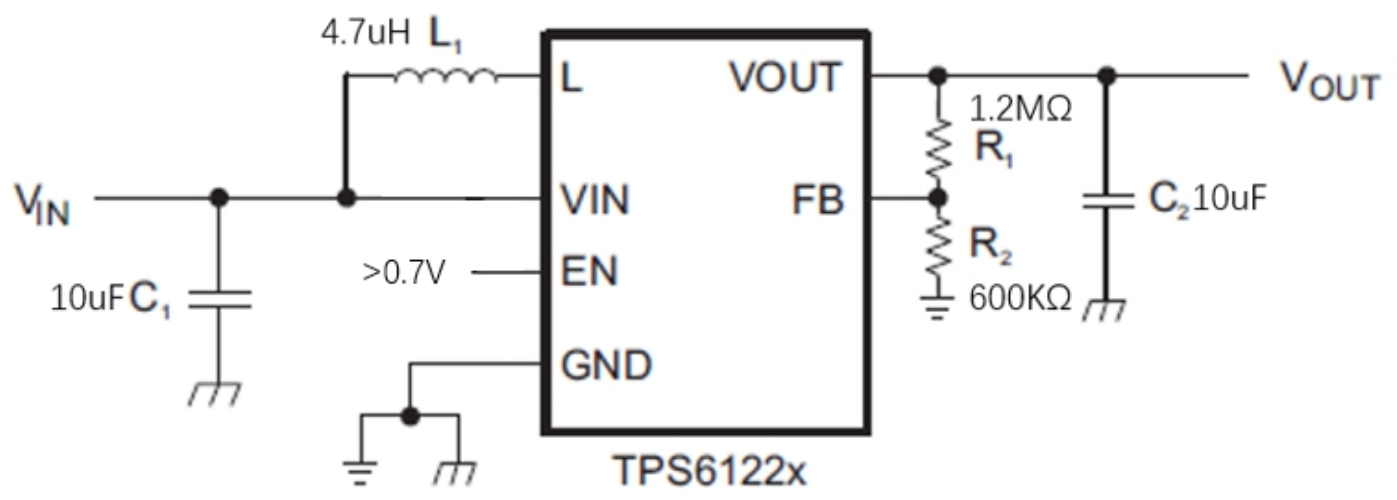

Fig 5. Circuit of boost module

b) Design of hysteresis comparator

A hysteresis comparator with upper and lower threshold voltage is formed as a result of a positive feedback network based on a single threshold voltage comparator ${ }^{[8]}$. Circuit of hysteresis comparator is depicted in Fig 6. R3 and R4 form the positive feedback network. R5 and R6 adjust the input comparison voltage. Zener diodes are employed for fixed output voltage and the output voltage should attain the enable voltage of boost module. Formulas of upper and lower threshold voltage are depicted in Eq 1 and Eq 2.

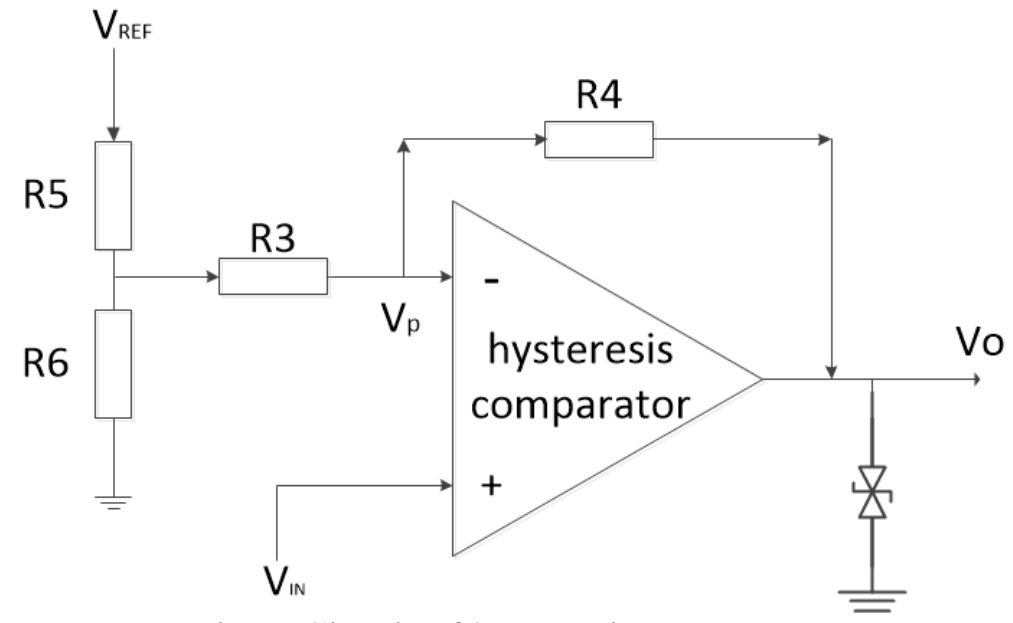

Fig 6. Circuit of hysteresis comparator

$$
\begin{gathered}
V_{T+}=\frac{R_{4} R_{6} V_{R E F}+R_{3} V_{O H}}{\left(R_{3}+R_{4}\right)\left(R_{5}+R_{6}\right)} \\
V_{T-}=\frac{R_{4} R_{6} V_{R E F}+R_{3} V_{O L}}{\left(R_{3}+R_{4}\right)\left(R_{5}+R_{6}\right)}
\end{gathered}
$$


2) Construction of experimental equipment

This paper provides special apparatus to test data of this charging method. As shown in the Fig 7, the experimental apparatus are mainly composed of a black box with variable light sources and voltage and current detecting instruments. Part 1 are the black box and a solar panel and a illuminometer are placed in it, the amount of electricity collected by the supercapacitor could be controlled through adjusting the brightness and light time of the top LED lamp. In this experiment, the intensity of light is 200LUX. Part 2 is the circuit using this method. Part 3 is the voltage and current testing device.

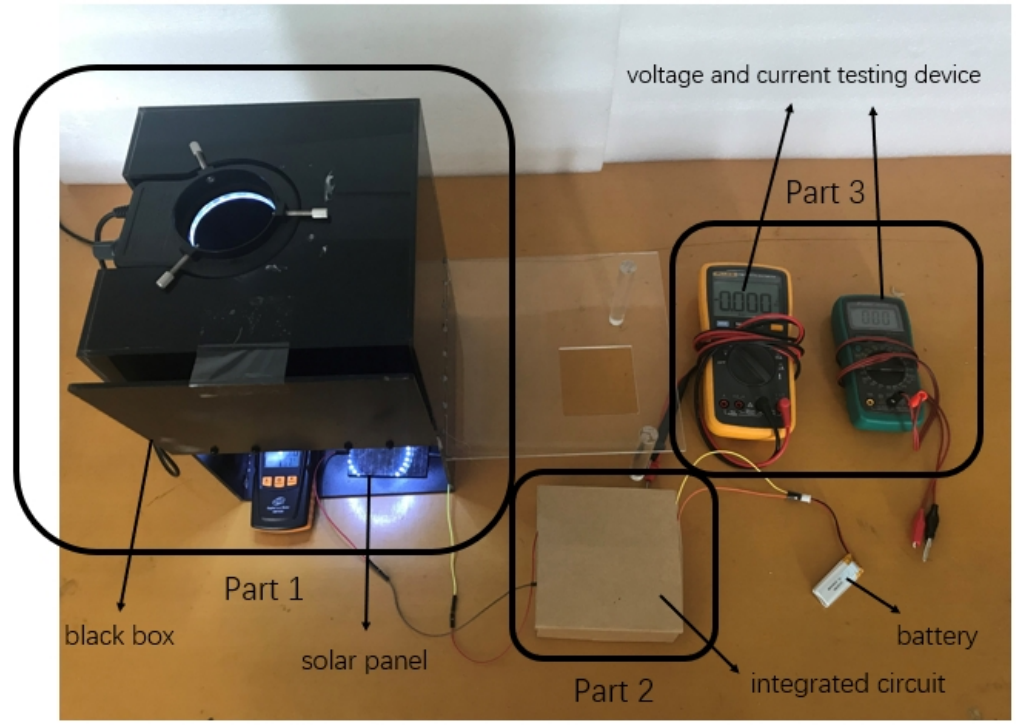

Fig 7. Construction of experimental equipment

3) Experimental data and analysis

a) Experiment of supercapacitor

Retaining supercapacitor and solar panel, the relationship between the voltage of supercapacitor and illumination time is measured by the experimental equipment under the 200LUX light intensity. The experimental results, as shown in Fig 8, show that the voltage shows a linear upward trend at the beginning of the energy storage but the trend gradually decreases, and eventually tends to be slow. It shows that the electric energy loss will occur when the supercapacitor has a high voltage.

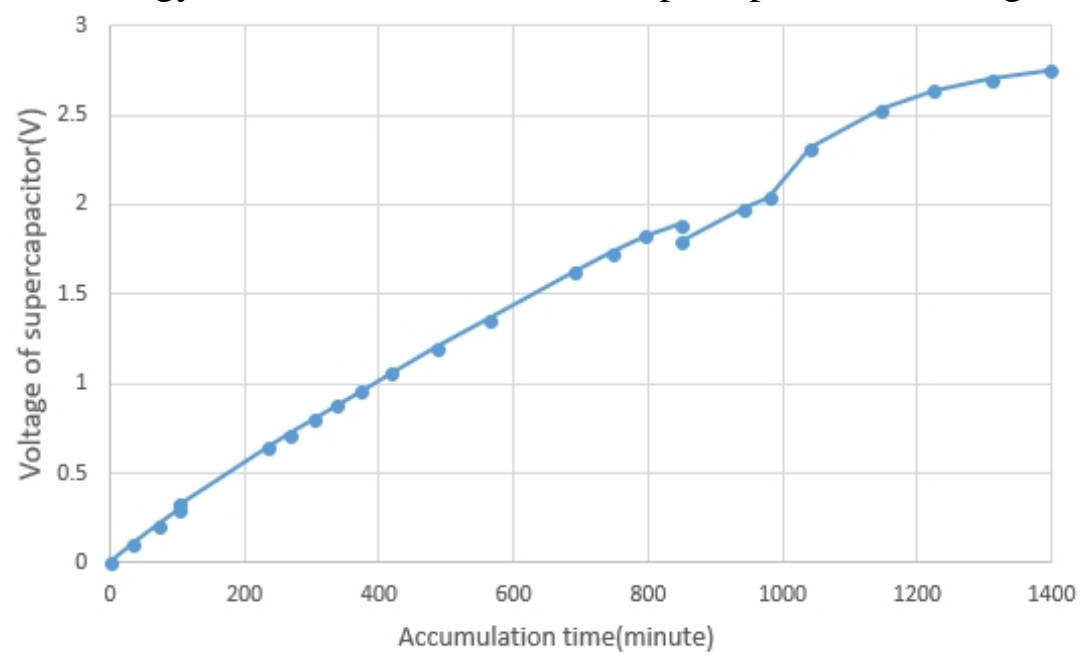

Fig 8. Experiment data of supercapacitor

b) Experiment of lower threshold voltage

The lower threshold voltage of the super capacitor of different lithium battery voltage is tested by the experimental apparatus and the experimental data is depicted in Fig 9. There is no obvious connection between the lower threshold voltage and the voltage of the battery. Because of the 
contingency of data, the recommended ratio between lower threshold voltage and voltage of battery is 0.3. Bring the ratio into Eq 2 and selecting $\mathrm{V}_{\mathrm{OH}}$, the formula of resistances will be attained.

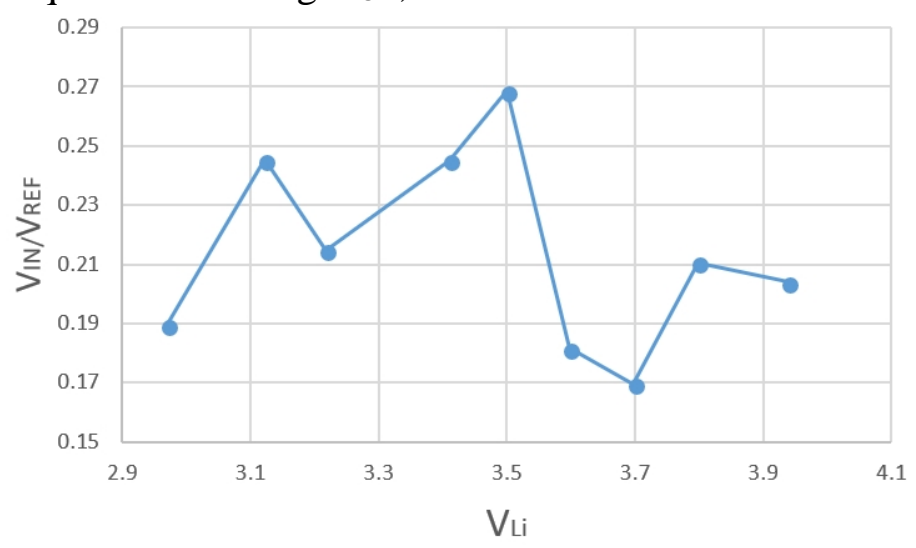

Fig 9. Experiment data of lower threshold voltage

c) Analysis and conjecture of upper threshold voltage

About upper threshold voltage, the power loss of supercapacitor should be taken into account and the loss will increase with the increase of supercapacitor voltage. When the voltage of the lithium battery is $3.65 \mathrm{~V}$, the supercapacitor discharge voltage of $1.1 \mathrm{~V}, 1.4 \mathrm{~V}, 1.8 \mathrm{~V}, 2.2 \mathrm{~V}, 2.6 \mathrm{~V}, 2.9 \mathrm{~V}$ was chosen for the experiment, corresponding to the ratio of the upper threshold voltage and the lithium battery $0.3,0.4,0.5,0.6,0.7,0.8$. The experimental results are as shown in Fig 10, the ordinate is the charging power into the lithium battery, and the abscissa is the storage time of the supercapacitor. Combined with Fig 8, it can be concluded that when the ratio is 0.6 to 0.7 , the power loss is small and the charging power is a reasonable value. It is speculated that the range between the upper threshold voltage and the lithium battery voltage recommended by this paper is 0.6 to 0.7 .



Fig 10. Upper threshold voltage experiment data

\section{Conclusions}

This paper presents an alternate charging method, where ASS is able to collect weak energy in low-light conditions. The proposed method provides a relatively high efficient charging mode to battery of ASS. By using supercapacitor and hysteresis comparator sufficient and appropriate electrical energy is provided. Moreover, by employing boost module and charging management module the electrical energy below the battery voltage is charged into the battery. In the experiment of recommended circuit in paper, the recommended ratio of lower threshold voltage (supercapacitor stop discharge voltage) to lithium battery voltage is 0.3 and the recommended ratio of upper threshold voltage (super capacitor discharge voltage) to lithium battery voltage is $0.6 \sim 0.7$. 


\section{Acknowledgements}

This work was financially supported by the Zhejiang Xinmiao Talent Plan (2017R415014).

\section{References}

[1] Wu Wei: submitted to China Semiconductor Lighting Promotion Project (2017)

[2] Guiqing Jiang, Research And Design Of Embedded Smart Home System, Master, Southwest Jiaotong University, Chengdu, Sichuan Province(2012)

[3] Qin Liu, Design of A Wireless Intelligent Switch With High Flexibility, Master, Kunming University Of Science And Technology, Kunming, Yunnan Province (2015)

[4] Lijun Wang, China Patent ZL 201721347466.4

[5] Jianming Chen: Technology And Market, Vol. 22(2015), p.24-25

[6] Information on http://www.ti.com.cn/product/cn/tps61221

[7] HE Qiu-sheng: submitted to Power Supply Technology(2013)

[8] Huaguang Kang: Electronic Technology Foundation(Higher Education Press, China( 2005) 